\title{
Animal Surveillance: Use of Animal Health Data to Improve Global Disease Surveillance
}

\author{
Karen L. Meidenbauer* \\ Johns Hopkins University APL, Columbia, MD, USA
}

\section{Objective}

To identify gaps in current U.S. animal data collection and surveillance systems, describe how surveillance of animal populations can provide important early warnings of emerging threats to human populations from infectious disease epidemics, and explain the benefits of integrating human and animal surveillance data into a common linked system.

\section{Introduction}

Since the majority of emerging infectious diseases over the past several decades have been zoonotic, animal health surveillance is now recognized as a key element in predicting public health risks. Surveillance of animal populations can provide important early warnings of emerging threats to human populations from bioterrorism or naturally occurring infectious disease epidemics. This study investigated current animal data collection and surveillance systems, isolated major gaps in state and national surveillance capabilities, and provided recommendations to fill those gaps.

\section{Methods}

Initially, an extensive literary review was performed to better understand what is currently available for Animal Health Disease Surveillance in the United States and recognize the gaps. After this review meetings were arranged with numerous animal health and public health surveillance experts to isolate their surveillance priorities: Department of Homeland Security (DHS), USDA Animal Plant Health Inspection Service (APHIS), U.S. Army Veterinary Corps, University Laboratories/Veterinary Teaching Hospitals, The National Capitol Region (NCR) ESSENCE Public Health Steering Committee, Maryland Arbovirus, Zoonotic, and Vector Disease Group, and the Maryland State Veterinarian.

A key animal disease surveillance stakeholder group that has been underrepresented in prior requirements assessments is private practitioners. Preliminary discussions with key practitioners revealed clearly that there are monumental gaps in animal health surveillance and it frequently limits their ability to rapidly respond to potential disease risks within their animal population of concern. To better understand these gaps and potential ways to improve surveillance in this area, a voluntary survey was developed and sent out to members of the Maryland Veterinary Medical Association, Virginia Veterinary Medical Association, and the District of Columbia Academy of Veterinary Medicine.

\section{Results}

Through this comprehensive study three current U.S. animal health disease surveillance gaps were isolated: integrated human and animal health surveillance, real-time animal health data collection, and companion animal surveillance.

The survey was also well received and had almost 160 participants. Key issues addressed in the survey included: Animal Medical Records - availability, capabilities, and concerns, Zoonotic disease exposure and reporting, and support for development of integrated humananimal disease surveillance tools.

Key Findings:
- Almost $90 \%$ of responding practitioners reported having encountered a zoonotic disease in practice.

- Although less than $50 \%$ have reported a zoonotic disease to the state or federal government.

- Almost $70 \%$ of veterinarians in the National Capital Region (NCR) who participated in the survey also reported that they do not have access to a surveillance system.

- Veterinarian's responses to the question: "What is your opinion of the current status of local, regional, or national zoonotic disease surveillance and the use of animal data for surveillance?":

"I think it is difficult to find up to date local and regional data. Email alerts etc. would be nice, rather than having to search for information that frequently isn't current."

"I feel that many zoonotic diseases go unreported due to the lack of ease of reporting them and there is no communication between the human and veterinary medical communities as far as reportable diseases affecting both people and animals."

"With the proliferation of tick borne disease, closer surveillance of animal cases would benefit human medicine. We knew exactly when Lyme hit our area. It was three years later before VA Dept. of Health sent out a letter outlining the prevalence of disease in southwest VA human cases."

\section{Conclusions}

Linking the systems that report human and animal diseases would enable health professionals to swiftly identify and respond to zoonotic disease outbreaks. Since funding for animal health surveillance is limited, integrating animal data into existing, well-established human health surveillance systems would reduce the resources needed while still providing the advanced capabilities that are available for human health surveillance. The need for integrated surveillance has been recognized by regulatory officials, but concerns regarding funding, data acquisition, data confidentiality, and identification of desired stakeholders must still be addressed. The sometimes disparate interests of large industry, private practitioners, and state governments make gaining access to large centralized pools of animal health data a challenge. By using existing human health surveillance systems as a platform to develop integrated human-animal surveillance systems and by working with experts in the human surveillance field, these concerns can be ameliorated. This would lead to more advanced integrated health surveillance capabilities and heighten the nation's ability to quickly detect and respond to emerging zoonotic diseases. 
The Ideal Integrated National Surveillance System Format:
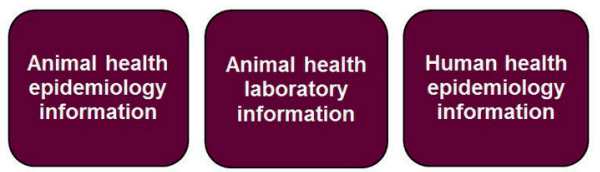

Human health

laboratory

information

Data visualization features for corroboration

Fusion algorithms looking for mathematical correlations

Enhanced Public Health Outcomes
Joint routine integrated qualitative risk assessment

\section{Keywords}

One Health; Integrated Surveillance; Animal Health; Public Health

\section{Acknowledgments}

Virginia-Maryland College of Veterinary Medicine, Virginia Polytechnic Institute and State University, and Johns Hopkins University Applied Physics Laboratory.

\section{*Karen L. Meidenbauer}

E-mail: karen.meidenbauer@jhuapl.edu 